

Original Research Article

\title{
Lactoperoxidase inhibition by tautomeric propylthiouracils
}

\author{
Mehdi Soleimania, Mahmoud Mirzaeib,*, Mohammad R. Mofidc, Ghadamali \\ Khodarahmia , S. Farid Rahimpoura \\ a Department of Medicinal Chemistry, School of Pharmacy and Pharmaceutical Sciences, Isfahan University of Medical Sciences, \\ Isfahan, Iran \\ b Department of Biomaterials, Nanotechnology and Tissue Engineering, School of Advanced Technologies in Medicine, Isfahan \\ University of Medical Sciences, Isfahan, Iran \\ c Department of Biochemistry, School of Pharmacy and Pharmaceutical Sciences, Isfahan University of Medical Sciences, \\ Isfahan, Iran
}

\section{ARTICLE INFORMATION}

Received: 15 January 2019

Received in revised: 20 January 2019

Accepted: 20 January 2019

Available online: 2 March 2019

DOI: $10.22034 / A J G C / 2020.1 .1$

\section{KEYWORDS}

\section{Propylthiouracil}

Tautomer

Lactoperoxidase

Molecular docking

Quantum computation

\begin{abstract}
Lactoperoxidase (LPO) enzyme inhibition by tautomeric propylthiouracil (PTU) structures have been investigated in this work based on the in silico methodologies. Six possible PTU structures have been optimized to obtain their energy-minimized structures based on quantum mechanics computations. Afterwards, their interactions with LPO enzyme have been evaluated based on molecular docking simulations. The results indicated that the structural changes of PTU analogues could perturbate the interaction properties, in which it could be seen by either the magnitudes of binding energies or the types of interacting amino acids. In this work, the original thio-keto structure of PTU showed better interaction properties with LPO enzyme; however, the properties for other PTU derivatives have been deviated from this reference model. It is known that the tautomerism is common for biological structures; therefore, exploring their arisen effects on the structural properties and activities could reveal insightful information for judging their potency and efficacy.

(C) 2020 by SPC (Sami Publishing Company), Asian Journal of Green Chemistry, Reproduction is permitted for noncommercial purposes.
\end{abstract}




\section{Graphical Abstract}



\section{Introduction}

Propylthiouracil (PTU, Scheme 1), which has been initially used in the 1940s, is a one of the most important medicines in the hyperthyroidism treatment [1]. Its function is based on decreasing the amount of thyroid hormone and preventing the conversion of thyroxine $\left(\mathrm{T}_{4}\right)$ to triiodothyronine $\left(\mathrm{T}_{3}\right)$ by inhibition of peroxidase enzymes [2]. Comparing with another medicine; methimazole, PTU has been considered as more effective in a thyrotoxic crisis [3]. Besides the advantages, PTU treatment shows side effects for the patients such as hair loss, swelling, muscle pains, numbness, and headache in addition to major problems with liver and blood cells [4]. Moreover, although PTU is recommended to be used during pregnancy, there could be harmful effects for the baby [5]. Therefore, it seems that further investigations are required to explore PTU analogues with better activities [6, 7].

The original PTU structure (Scheme 1), one of the pyrimidine uracil nucleobase derivatives, includes thio and keto-functional groups, in which both are good candidates to participate in the tautomerism as a common process in the organic compounds [8-10].

Tautomerism is one of the major reasons for occurrence of mutation in biological molecules, in which it could change the activity of tautomerized structure in comparison to the desired original structure [11]. It is widely known that the specific structure dictates the specific activity for the chemical/or medicinal compound thorough structure-activity relationship (SAR) [12]. Therefore, careful examination of structures could reveal insightful information about the observed activities for a specified chemical/or medicinal compound.

Within this work, formations of PTU tautomers and their corresponding activities have been investigated based on quantum chemical computations and molecular docking simulations by the advantage of employing in silico methodologies for exploring the chemicals in the molecular/or atomic scales. To this aim, we have primarily evaluated physico-chemical properties which have been 
initially evaluated for each of the tautomeric structures; then, their interactions with lactoperoxidase (LPO) enzyme have been examined, as a representative of contributing peroxidase enzymes to hyperthyroidism [13]. In an earlier work, Freeman and Po [14] reported the tautomerism of 2pyrimidinethiol and 2(1H)-pyrimidinethione to show the arisen significant effects on the corresponding structural properties. Recently, Palafox and co-worker [15] indicated the importance of sulfur atomic situation in determining the interactive properties of thiouracil derivatives.

Scheme 1. Propylthiouracil (PTU)



Accordingly, both of arisen intra-molecular (structural) and inter-molecular (activity) effects of PTU tautomers have been investigated in the current work by combinations of pure quantum chemical computations and molecular docking simulations

\section{Experimental}

\section{Computational details}

Tautomers of PTU (ChemSpider ID: 571424) have been constructed by movements of hydrogen atoms of pyrimidine nitrogen (N1 and N3) atoms between sulfur (S2) and oxygen (O4) atoms resulting five tautomers in addition to the original thio-keto from; L12-L34, in which the 2-digit numbers indicate the position of $H$ atoms on the PTU atomic sites. L13 is the original thio-keto form, L12 and L23 are the tautomeric thiol-keto forms, L14 and L34 are the tautomeric thio-enol forms, and L24 is the tautomeric thiol-enol form. All six structures have been optimized by the B3LYP/6$311++G^{* *}$ density functional theory (DFT) methodology as implemented in the Gaussian package [16-19] to evaluate the minimized-energy geometries for further electronic investigations. Molecular properties including total energy difference $\left(\Delta E_{\mathrm{T}}\right)$, energies of the highest occupied, the lowest unoccupied molecular orbital and their differences ( $E_{\text {номо }}, E_{\mathrm{LUMO}}$ and $\left.E_{\mathrm{G}}\right)$, dipole moment $\left(D_{\mathrm{M}}\right)$, ionization potential $(I)$, electron affinity $(A)$, chemical hardness $(\eta)$, chemical softness $(\sigma)$, octanolwater partition coefficient $(\log \mathrm{P})$, and molecular volume $(V)$. The latter two parameters; $\log \mathrm{P}$ and $V$ have been evaluated by the HyperChem package [20], and the procedure of evaluation of other parameters could be achieved with an earlier work [21]. Time-dependent (TD) calculations at the mentioned DFT level have been also performed to evaluate the HOMO and LUMO distribution patterns. All results are included in Table 1. 
Subsequently, in silico molecular docking simulations have been performed employing the genetic algorithm (GA) as implemented in the AutoDock package [22-25]. The 3D structure of LPO (lactoperoxidase) enzyme, as a representative of peroxidase enzymes in hyperthyroidism, has been obtained from PDB (ID: 5HPW) and its chain A has been prepared for the docking process. The size of grid box has been set to $70 * 70 * 70$ and the number of run seeds has been set to 300 for all of molecular docking simulations. The obtained results including binding energy $\left(E_{\mathrm{B}}\right)$, inhibition constant $\left(K_{\mathrm{i}}\right)$, oxygen and haem iron (O-Fe) distance, the contributing amino acids of LPO to hydrogen bonding (HB) and non-HB interactions (Table 2). The visual representations of LPO-PTU interactions have been also prepared by discovery studio package (Scheme 2).

Table 1. Molecular descriptors for PTUsa

Model
Figures


a L13 is the original PTU and other Ls are the tautomers

\section{Results and Discussion}

The evaluated molecular descriptors by optimizations of models of this work including the original thio-keto (L13) structure of PTU (Scheme 1) and all other possible tautomeric thiol-keto (L12 and L23), thio-enol (L14 and L34), and thiol-enol (L24) structures are presented in Table 1. It is remembered that the 2-digit numbers indicate the positions of $H$ atoms on the atomic sites of each L structure of PTU; e.g., atoms number 1 and 3 hold $H$ atoms in L13. The optimized energies indicate that the original L13 is the most stable structure among all the models; L23 is the most and L34 is the least stable tautomer of PTU. The energy barriers, as indicated by $\Delta E_{\mathrm{T}}$, for conversion of original L13 to other possible tautomers are less than $1 \mathrm{eV}$ meaning the possibility of easy formation of tautomers;

Table 2. Molecular docking properties for PTUs and enzymea

$\begin{array}{ccccccc}\text { Model } & \text { L12 } & \text { L13 Original } & \text { L14 } & \text { L23 } & \text { L24 } & \text { L34 } \\ E_{\mathrm{B}}(\mathrm{kcal} / \mathrm{mol}) & -5.85 & -5.87 & -5.83 & -5.71 & -5.78 & -5.79 \\ K_{\mathrm{i}}(\mu \mathrm{M}) & 49.98 & 49.66 & 53.56 & 65.47 & 58.31 & 56.99 \\ \text { O-Fe Distance }(\AA) & 2.84 & 2.74 & 3.03 & 9.81 & 3.01 & 2.81 \\ \text { HB- Interactions } & \text { GLN105 } & \text { HAEM } & \text { HAEM } & \text { PHE422 } & \text { HAEM } & \text { HAEM } \\ & & \text { GLN105 } & \text { GLN105 } & \text { GLN423 } & \text { GLN105 } & \text { HIS109 } \\ & & \text { ARG255 } & \text { HIS109 } & & \text { HIS109 } & \text { ARG255 } \\ \text { Non-HB-Interactions } & \text { HAEM } & \text { HAEM } & \text { HAEM } & \text { HAEM } & \text { HAEM } & \text { HAEM } \\ & \text { HIS109 } & \text { HIS109 } & \text { PHE113 } & \text { GLN105 } & \text { PHE113 } & \text { GLN105 } \\ & \text { PHE113 } & \text { PHE113 } & \text { ARG255 } & \text { HIS109 } & \text { ARG255 } & \text { PHE113 } \\ & \text { ARG255 } & \text { ARG255 } & \text { GLU258 } & \text { ARG255 } & \text { GLU258 } & \text { ARG255 } \\ & \text { GLU258 } & \text { GLU258 } & \text { PHE381 } & \text { GLU258 } & \text { PHE381 } & \text { GLU258 } \\ & \text { PHE381 } & \text { PHE381 } & \text { PRO424 } & \text { PHE380 } & \text { PRO424 } & \text { PHE381 } \\ & \text { PR0424 } & \text { PR0424 } & & \text { PHE381 } & & \text { PR0424 }\end{array}$

a See Scheme 2 for visual information

very much possible for $\mathrm{L} 13 \rightarrow \mathrm{L} 23$. Then, the results indicate that although the thio-keto structure is the most favorable one based on stabilized energies; however, its stability could be broken under an energy resource such as sunshine, warmness, etc. The HOMO-LUMO distribution patterns and their 
corresponding energy levels also exhibit that the electronic environments of molecular orbitals detected the effects of tautomerization in the investigated PTU models. Since the molecular orbitals characters are dominant for arising the initial properties of matters, their variances from a reference point could mean their variances from a reference activity. Accordingly, assigning the values of $E_{\mathrm{G}}$ defined as the energy differences of HOMO-LUMO levels, to the reactivity property, we could mention that the highest unfavorable reactivity belongs to the earlier signified lowest stable tautomer: L34. It is important to note that the magnitudes of HOMO and LUMO energy levels are important to observe the reactivity properties, in which it could be seen that the magnitudes are different among the models. The originated electronic properties usually support each other, in which the largest magnitude of $D_{\mathrm{M}}$ (Dipole moment) belongs to the lowest stable structure; 8.38 for L34. Again coming back to the molecular orbitals, the observed tendency of PTU models to release or obtain electrons by the evaluated I (Ionization potential) and A (Electron affinity) properties show that the tautomeric process significantly change these characters in comparison with the original thio-keto model. Herein, we could refer to the importance of correspondence structural properties for efficient activities, in which the variations of I and A could significantly change the expected efficacy of PTU models. One of the major problems with the medicinal compounds is their unwanted side effects. On the other hand, tautomerization is a very common process for biological structures especially in the watery systems, which significantly change the structural properties. In this case, the magnitudes of $\eta$ (Chemical harness) and $\sigma$ (Chemical softness) could be compared with those of $E_{\mathrm{G}}$, in which the lowest $\eta$ and the highest $\sigma$ belong to the earlier low-stability detected structure: L34. Moreover, comparing the magnitude of $\log \mathrm{P}$, as the solubility of a compound in octanol-water solvents, shows that the water solubility is strongly decreased in the tautomeric structures versus the original L13. The magnitudes of molecular volume $(V)$ show the effects of tautomerization on the occupied space of PTU models, in which this space is also changed during the tautomerism.

As a brief conclusion of this part, it could be mentioned that the extended molecular descriptors could greatly help to analyze the structural properties in the lowest possible molecular/atomic scales. As an advantage of pure quantum chemical computations; the molecular descriptors could be obtained at the high accuracy and validity referred to the theoretical backgrounds and evidences. As a more specific trend about the investigated PTUs, the tautomerism significantly changes the original structural properties to new properties for the tautomers. The closed structural formulas for all original and tautomers are identical; however, their extended formulas show significant differences in comparison with each other. As a final remark, prevention of the thio-keto of PTU not to contribute to tautomerism could be considered as a crucial step to lead the structure to show desired activity. 
The intra-molecular structural properties for the single-standing models of original and tautomeric PTUs have been described above and the results indicated that the variations of structural properties among tautomerism. In this part, the inter-molecular properties of PTUs would be discussed based on their interactions with the LPO (Laxtoperoxidase) enzyme to explore the effects of tautomerism on the PTU activity. To this aim, molecular docking simulations have been performed to evaluate the inter-molecular properties for the PTU counterparts (Table 2 and Scheme 2). One of the most important docking parameters is $E_{\mathrm{B}}$ (Binding energy), which shows the magnitude of released energy through interaction process. Comparing the results indicates that L13 is the strongest interacting counterpart with the enzyme among other PTU models. $K_{\mathrm{i}}$ (Inhibition constant) is another complementary docking parameter, in which its direction is reversed versus $E_{\mathrm{B}}$ meaning increasing the net value of $E_{\mathrm{B}}$ is followed by decreasing the value of $K_{\mathrm{i}}$. The major point of these comparisons of $E_{\mathrm{B}}$ and $K_{\mathrm{i}}$ for the investigated PTU models is that the tautomerism is not suitable for

\section{E-L12}

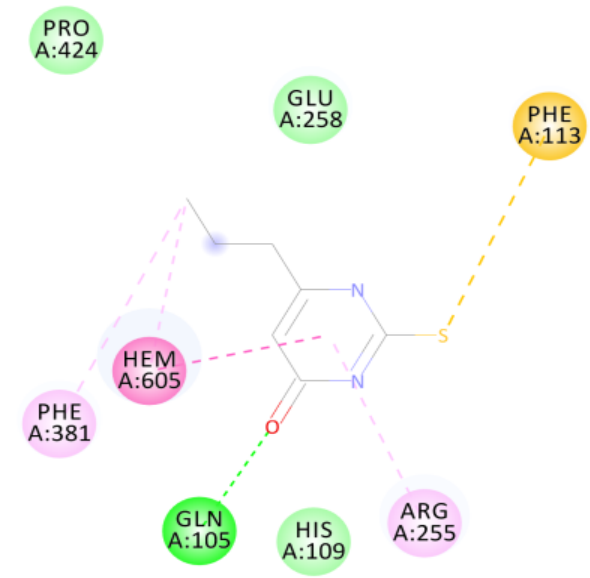

E-L14

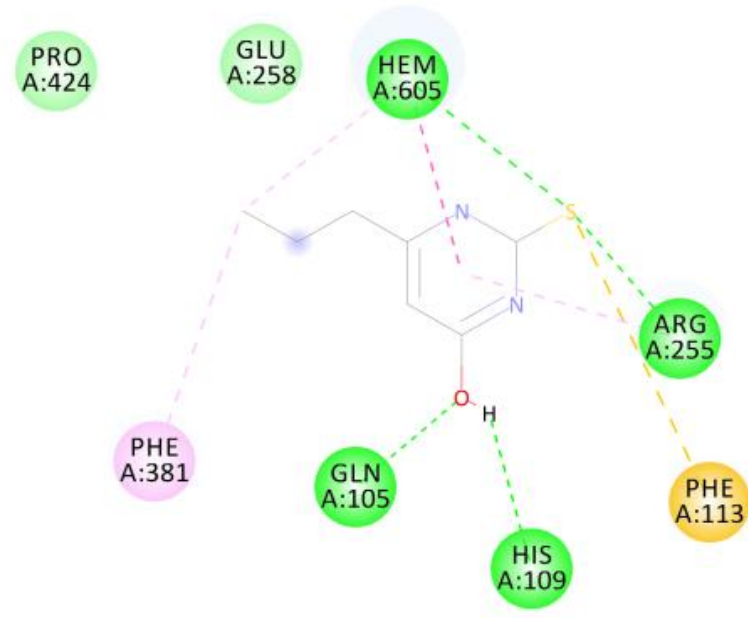

\section{E-L13 Original}

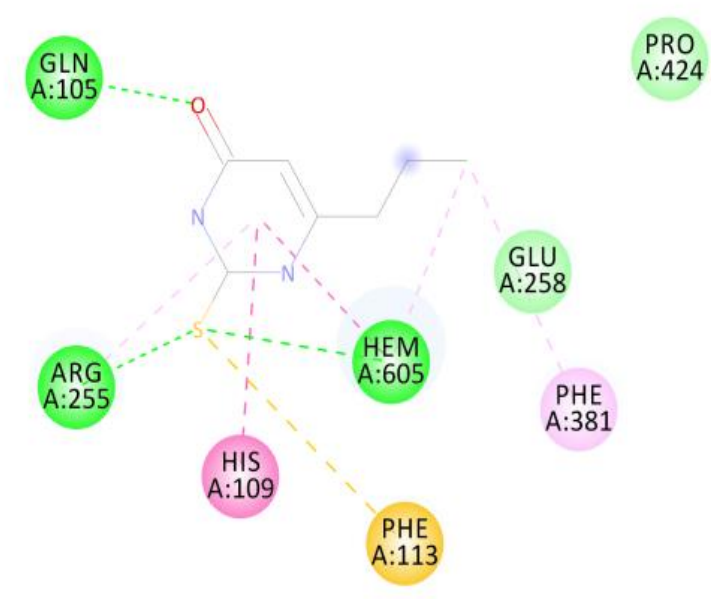

E-L23

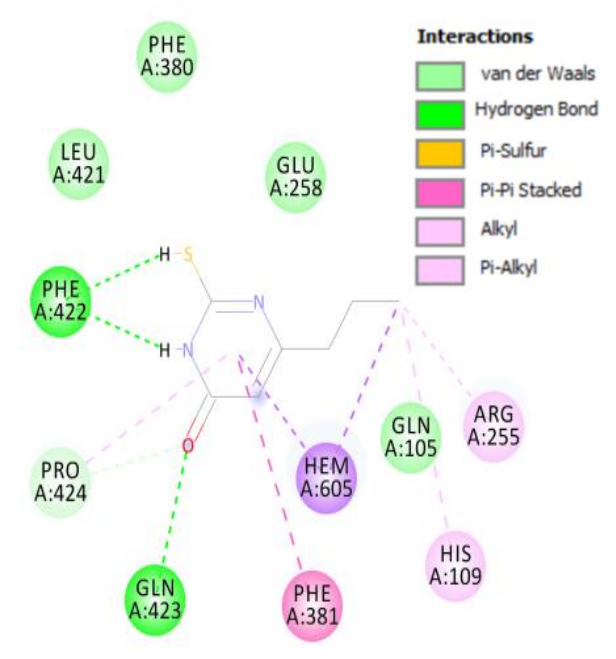




\section{E-L24}

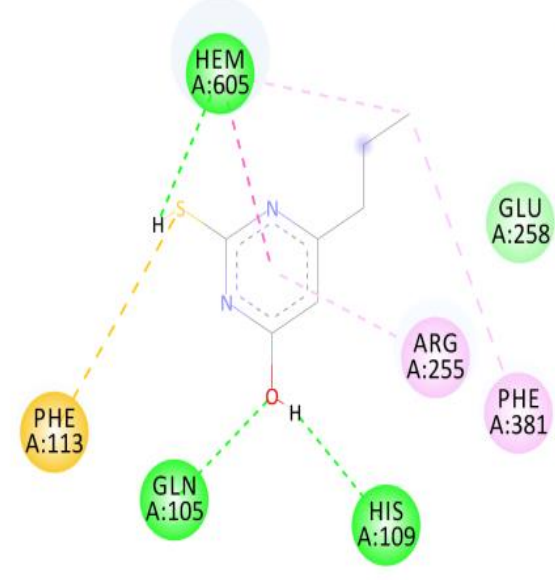

E-L34



PRO
A:424

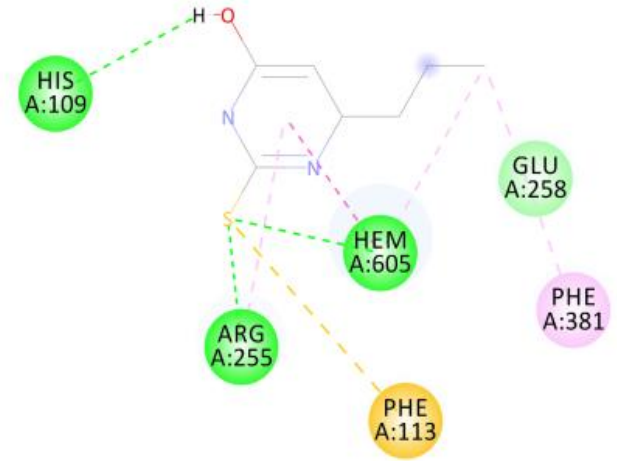

PRO

$A: 424$

See Table 2 for details

Scheme 2. Visual representations of enzyme-PTU (E-L) interactions*

PTU efficacy and it is better to be prevented by structural modifications, etc. Interestingly, the shortest distance of $O$ atom of carbonyl group with Fe of haem group of enzyme belongs to L13 approving the efficacy of thio-keto PTU for proper binding to LPO enzyme. Amazing result is for L23, in which the PTU position has been shifted to farther site of major interactions. As an importance of haem group existence in the active site of LPO enzyme, the interaction of PTU to haem group is crucial as could be seen by the most favorable interaction distance between original PTU with the haem group in correspondence with the largest $E_{\mathrm{B}}$ magnitude among the models. Examining the situations of HB (hydrogen bond) interactions could reveal that the amino acid counterparts are almost changed in different PTU involving systems but more similarities could be seen for the non-HB interactions.

As a brief conclusion of this part, it could be mentioned that the inter-molecular properties of PTU models are changed during the tautomerism process. Although the variances of $E_{\mathrm{B}}$ values are not very much significant, less than $0.5 \mathrm{kcal} / \mathrm{mol}$, their interacting environments with amino acids are remarkably changed from one tautomer to another or to original model. Moreover, the magnitudes of $K_{\mathrm{i}}$ also undergo more significant changes in comparison to the original PTU; L13. The haem group is located in the active site of enzyme, but it has been seen that L23 does not interact with this important group. Remembering the structural properties of single-standing PTUs could support the evaluated results of molecular docking simulations, in which it was expected that the changes of structural properties could change the corresponding activities for the specific compounds. Moreover, clarifying the details of both structural and activity properties of PTU could reveal insightful information about the hidden mystery under the face of its closed formula. 


\section{Conclusion}

Within this work, properties of PTU tautomers and their corresponding interacting activities have been investigated based on the in silico methodologies. Our results indicated that the effects of tautomerism have been observed for the tautomeric structures of PTU by the evaluated molecular descriptors. Moreover, the thio-keto model of PTU has been seen as the most stable structure among the possible tautomers; however, its conversion to tautomers could be possible. Interestingly, both of the binding energies and amino acid types have been changed in the interactions of PTU tautomers and LPO enzyme. As a final remark, the activity of original thio-keto structure is changed during tautomerism process, which could be claimed as a point for arising side effects.

\section{Acknowledgements}

The financial support of this work by the research council of Isfahan University of Medical Sciences (Grant No. 396672) is acknowledged.

\section{Disclosure Statement}

No potential conflict of interest was reported by the authors.

\section{References}

[1]. McCormick R.V.J. Am. Med. Ass., 1950, 144:1453

[2]. Hamajima E., Noda M., Nai E., Akiyama S., Ikuta Y., Obana N., Kawaguchi T., Hayashi K., Oba K., Yoshida T., Katori T., Kokaji M. Clin. Ped. Endoc., 2018, 27:171

[3]. Zeng X., Zhan L., Zhao Y. Int. J. Clin. Exp. Med., 2018, 11:2642

[4]. Shaikh H.G., Kamran A., Mewawalla P. Blood, 2017, 130:4959

[5]. Andersen S.L., Olsen J., Wu C.S., Laurberg P. Thyroid, 2014, 24:1533

[6]. Chen H., Zhang X., Jia X., Liu Z. Regul. Toxicol. Pharmacol., 2018, 97:120

[7]. Budzák Š., Mach P., Juhász G., Medved M., Kysel O. Comput. Theor. Chem. 2015, 1051:129

[8]. Yaraghi A., Ozkendir O.M., Mirzaei M. Superlatt. Microstruct., 2015, 85:784

[9]. Mirzaei M. J. Turk. Comput. Theor. Chem., 2017, 1:27

[10]. Colasurdo D.D, Pila M.N., Iglesias D.A., Laurella S.L., Ruiz D.L. Eur. J. Mass Spectrom., 2018, 24:214 [11]. Raczyńska E.D., Sapuła M., Zientara-Rytter K., Kolczyńska K., Stępniewski T.M., Hallmann M. Struct. Chem., 2016, 27:133

[12]. Rosenberg S.A., Watt E.D., Judson R.S., Simmons S.O., Friedman K.P., Dybdahl M., Nikolov N.G., Wedebye E.B. Comput. Toxicol., 2017, 4:11 
[13]. Singh P.K., Sirohi H.V., Iqbal N., Tiwari P., Kaur P., Sharma S., Singh T.P. Biochim. Biophys. Acta, 2017, 1865:329

[14]. Freeman F., Po H.N. J. Phys. Chem. A, 2006, 110:7904

[15]. Palafox M.A., Rastogi V.K., Singh S.P. J. Biomolec. Struct. Dyn., 2018, 36:1225

[16]. Frisch M.J., Trucks G.W., Schlegel H.B., Scuseria G.E., Robb M.A., Cheeseman J.R., et al. Gaussian 09, Revision A.01, Gaussian Inc., Wallingford, CT, 2009

[17]. Mirzaei M., Elmi F., Hadipour N.L. J. Phys. Chem. B, 2006, 110:10991

[18]. Mirzaei M., Kalhor H.R., Hadipour N.L. J. Mol. Model., 2011, 17:695

[19]. Amirabadi A.H.R., Mirzaei M. Iran. Chem. Commun., 2019, 7:223

[20]. HyperChem (TM) Professional 8.0.3, Hypercube Inc., Gainesville, FL, 2007

[21]. Aramideh M., Mirzaei M., Khodarahmi G., Gülseren O. Z. Naturforsch. A, 2017, 72:1131

[22]. Morris G.M., Huey R., Lindstrom W., Sanner M.F., Belew R.K., Goodsell D.S., Olson A.J. J. Comput. Chem., 2009, 16:2785

[23]. Shahpar M., Esmaeilpoor S. Asian J. Green Chem., 2017, 1:116

[24]. Fazlinezhad M., Nakhaei A., Eshghi H., Saadatmandzadeh M. Iran. Chem. Commun. 2019, 7:125

[25]. Bommeraa R.K., Merugu R., Eppakayala L. Chem. Method., 2019, 3:354

How to cite this manuscript: Mehdi Soleimani, Mahmoud Mirzaei, Mohammad R. Mofid, Ghadamali Khodarahmi, S. Farid Rahimpour. Lactoperoxidase inhibition by tautomeric propylthiouracils. Asian Journal of Green Chemistry, 4(1) 2020, 1-10. DOI: 10.22034/AJGC/2020.1.1 